\title{
Phase 2 study of efgartigimod, a novel FcRn antagonist, in adult patients with primary immune thrombocytopenia
}

\author{
Adrian C. Newland ${ }^{1}$ | Blanca Sánchez-González ${ }^{2}$ | László Rejtő ${ }^{3}$ | Miklos Egyed ${ }^{4}$ | \\ Nataliya Romanyuk $^{5}$ | Marie Godar ${ }^{6}$ (1) | Katrien Verschueren ${ }^{6}$ | Domenica Gandini ${ }^{6}$ | \\ Peter Ulrichts $^{6}$ | Jon Beauchamp ${ }^{6}$ | Torsten Dreier ${ }^{6}$ | E. Sally Ward ${ }^{7,8}$ ( ) \\ Marc Michel $^{9}$ | Howard A. Liebman ${ }^{10}$ | Hans de Haard ${ }^{6}{ }^{\infty}$ | Nicolas Leupin ${ }^{6}$ | \\ David J. Kuter ${ }^{11}$ (ㅇ
}

${ }^{1}$ Department of Haematology, Centre for Haematology, The Royal London Hospital, London, UK

${ }^{2}$ Department of Hematology, Hospital del Mar, Barcelona, Spain

${ }^{3}$ Department of Hematology, Jósa András Teaching Hospital, Nyíregyháza, Hungary

${ }^{4}$ Department of Hematology, Kaposi Mor Teaching Hospital, Kaposvar, Hungary

${ }^{5}$ Mykolaiv Regional Clinical Hospital, Mykolaiv, Ukraine

6argenx BVBA, Industriepark-Zwijnaarde 7,

Zwijnaarde, Belgium

${ }^{7}$ Department of Molecular and Cellular Medicine, Texas A\&M University Health Science Center, College Station, Texas

${ }^{8}$ Centre for Cancer Immunology, University of Southampton, Southampton, UK

${ }^{9}$ Service de Médecine Interne, Centre National de Référence des Cytopénies Auto-Immunes de l'Adulte, Centre Hospitalier Universitaire HenriMondor, Assistance Publique-Hôpitaux de Paris, Université Paris Est Créteil, Créteil, France

${ }^{10} J a n e$ Anne Nohl Division of Hematology, Department of Medicine, University of Southern California, Los Angeles, California

${ }^{11}$ Department of Hematology, Massachusetts General Hospital and Harvard Medical School, Boston, Massachusetts

\section{Correspondence}

Adrian C. Newland, Barts Health NHS trust The Royal London Hospital-Pathology Pharmacy Building 80 Newark Street, Whitechapel, London E1 2ES, UK. Email: a.c.newland@qmul.ac.uk

\begin{abstract}
Primary immune thrombocytopenia (ITP) is an acquired autoimmune bleeding disorder, characterized by a low platelet count $\left(<100 \times 10^{9} / L\right)$ in the absence of other causes associated with thrombocytopenia. In most patients, IgG autoantibodies directed against platelet receptors can be detected. They accelerate platelet clearance and destruction, inhibit platelet production, and impair platelet function, resulting in increased risk of bleeding and impaired quality of life. Efgartigimod is a human $\lg \mathrm{G} 1$ antibody Fc-fragment, a natural ligand of the neonatal Fc receptor ( $F C R n)$, engineered for increased affinity to FcRn, while preserving its characteristic $\mathrm{pH}$-dependent binding. Efgartigimod blocks FcRn, preventing IgG recycling, and causing targeted IgG degradation. In this Phase 2 study, 38 patients were randomized 1:1:1 to receive four weekly intravenous infusions of either placebo $(N=12)$ or efgartigimod at a dose of $5 \mathrm{mg} / \mathrm{kg}(\mathrm{N}=13)$ or $10 \mathrm{mg} / \mathrm{kg}(\mathrm{N}=13)$. This short treatment cycle of efgartigimod in patients with ITP, predominantly refractory to previous lines of therapy, was shown to be well tolerated, and demonstrated a favorable safety profile consistent with Phase 1 data. Efgartigimod induced a rapid reduction of total IgG levels (up to $63.7 \%$ mean change from baseline), which was associated with clinically relevant increases in platelet counts ( $46 \%$ patients on efgartigimod vs $25 \%$ on placebo achieved a platelet count of $\geq 50 \times 10^{9} / \mathrm{L}$ on at least two occasions, and $38 \%$ vs $0 \%$ achieved $\geq 50 \times 10^{9} / \mathrm{L}$ for at least 10 cumulative days), and a reduced proportion of patients with bleeding. Taken together, these data warrant further evaluation of FcRn antagonism as a novel therapeutic approach in ITP.
\end{abstract}




\section{1 | INTRODUCTION}

Primary immune thrombocytopenia (ITP) is an acquired autoimmune bleeding disorder characterized by a low platelet count $\left(<100 \times 10^{9} / \mathrm{L}\right)$ in the absence of other causes or disorders associated with thrombocytopenia. ${ }^{1-3}$ The low platelet count increases the risk of skin and mucosal bleeding, gastrointestinal bleeding complications and rarely, serious intracranial hemorrhages. ${ }^{2,4,5}$ Patients may suffer from depression and fatigue ${ }^{6}$ as well as side effects of existing therapies, impairing their quality of life. ${ }^{7-12}$ Current therapeutic approaches include non-specific immunosuppression (eg, steroids and rituximab), inhibition of platelet clearance (eg, splenectomy, intravenous immunoglobulin [IVIg], anti-D globulin, and the recently FDA-approved Syk inhibitor fostamatinib ${ }^{13}$ ) or stimulation of platelet production (eg, thrombopoietin receptor agonist [TPO-RA]). ${ }^{4,14}$ Splenectomy remains the only treatment that provides sustained remission off therapy for one year or longer for a high proportion of patients. ${ }^{3}$

Autoantibodies in ITP, which are predominantly of the IgG class, mediate pathogenic actions by targeting surface glycoproteins (GP) expressed on platelets and megakaryocytes, the progenitor cells of platelets. ${ }^{15,16}$ Detectable in most patients, they can opsonize platelets, resulting in clearance by splenic macrophages, induce platelet apoptosis, ${ }^{17}$ complement-dependent lysis ${ }^{18}$ or desialylation of platelets, and Fc-independent liver clearance. ${ }^{19}$ Moreover, they can inhibit megakaryocyte proliferation and differentiation resulting in diminished platelet production. ${ }^{20-22}$ Recently, it has been reported that some anti-GP antibodies interfere with platelet functionality, inhibiting platelet aggregation ${ }^{23}$ and blood clot formation. ${ }^{24}$ The majority of antiplatelet antibodies is directed against GPIIb/IIla and GPIb/IX, ${ }^{25,26}$ but additional targets have been identified. ${ }^{14}$ The central role of autoantibodies in the pathogenesis is further illustrated by occurrence of ITP in infants born to mothers with ITP, due to placental transfer of autoantibodies, ${ }^{27}$ and by historical use of IgG-depleting treatments like immunoadsorption and plasmapheresis, which lead to a reduction of platelet-associated autoantibodies $^{28}$ and increased platelet count. ${ }^{29}$

The neonatal Fc receptor ( $\mathrm{FcRn}$ ) is the central regulator of $\mathrm{lgG}$ homeostasis, rescuing IgGs from lysosomal degradation, prolonging IgG half-life, and promoting tissue distribution of $\operatorname{lgGs}{ }^{30,31}$ Albumin is also recycled by $\mathrm{FcRn}$, but binds at a site distinct from that of $\operatorname{lgGs}{ }^{32}$ Efgartigimod is a human IgG1 antibody Fc-fragment. ${ }^{33}$ This natural ligand of FcRn has been engineered with ABDEG mutations, located in the $\mathrm{CH} 2$ and $\mathrm{CH} 3$ domain of the Fc fragment to increase affinity for FcRn whilst preserving its characteristic $\mathrm{pH}$-dependent binding. Due to its increased affinity for $\mathrm{FcRn}$ at both acidic and neutral $\mathrm{pH}$, efgartigimod outcompetes IgGs for binding to FcRn, resulting in accelerated degradation of endogenous IgGs. ${ }^{30,34,35}$ In healthy volunteers (NCT03457649), efgartigimod was well tolerated and induced a rapid reduction of total lgGs and all lgG subtypes. ${ }^{33}$ A Phase 2 study in patients with myasthenia gravis, an IgG autoantibody-mediated neuromuscular condition (NCT02965573), showed similar tolerability, and IgG reduction associated with clinically and statistically significant improvements on efficacy scales. ${ }^{36}$
Targeted reduction of autoantibodies through FcRn blockade may prevent their pathogenic actions and represents a novel treatment modality in ITP. We investigated the safety and efficacy of efgartigimod in adult patients with primary ITP in a randomized, double-blinded, placebo-controlled Phase 2 study (NCT03102593).

\section{2 | METHODS}

\section{1 | Study design and treatment intervention}

In this randomized, double-blinded, placebo-controlled Phase 2 study (Figure S1), patients were randomized 1:1:1 to receive four weekly doses of either placebo or efgartigimod, at a dose of $5 \mathrm{mg} / \mathrm{kg}$ or $10 \mathrm{mg} / \mathrm{kg}$ body weight administered as an intravenous infusion. Patients were followed for up to 21 weeks (an initial eight-week period extended to 21 weeks after protocol amendment, Figure S1). After an additional protocol amendment, implemented part way through the study, patients who had a relapse during the 21-week follow-up period, defined as platelet count below $30 \times 10^{9} / \mathrm{L}$, had an option to enroll in an open-label treatment period where they received four weekly intravenous infusions of efgartigimod at $10 \mathrm{mg} / \mathrm{kg}$ (while maintaining the blind for the initial treatment allocation). The study was conducted in accordance with the Good Clinical Practice guidelines, in conformity with the ethical principles of the Declaration of Helsinki, was compliant with all relevant country-specific laws and regulations, and monitored by an Independent Data Monitoring Committee. The study protocol and all other appropriate study-related information were reviewed and approved by the independent ethics committees or institutional review boards.

\section{2 | Patients}

Thirty-eight patients were randomized in 19 study centers in Ukraine and seven countries in Europe. The study included patients aged 18 to 85 years, with confirmed primary ITP according to the American Society of Hematology guidelines, ${ }^{3}$ and an average of two platelet count measurements during the screening $<30 \times 10^{9} / \mathrm{L}$ (with no single reading $>35 \times 10^{9} / \mathrm{L}$ ). Concurrent ITP therapy (ie, oral corticosteroids, oral immunosuppressants, and/or TPO-RA) was permitted during the study, had to be on a stable dose and dosing frequency for at least four weeks prior to screening, and maintained during the study. Additionally, patients with total $\operatorname{lgG}$ level $<6 \mathrm{~g} / \mathrm{L}$ at screening were excluded. The presence of antiplatelet antibodies was not an inclusion criterion. All patients provided written informed consent prior to the commencement of any study-related procedures.

\section{3 | Safety and efficacy assessments}

The primary outcome was safety, assessed throughout the course of the study, including vital signs, electrocardiogram parameters, physical examination abnormalities, and clinical laboratory assessments. Treatment emergent adverse events (TEAEs) were coded according to the Medical Dictionary for Regulatory Authorities version 19.1. Secondary outcomes included platelet count responses and bleeding 
assessments. Other outcomes were the evaluation of the pharmacodynamic (PD) and pharmacokinetic parameters (PK), and immunogenicity. Measurements of circulating and platelet-bound autoantibodies were performed at Sanquin Diagnostic Laboratory using a commercially available solid-phase ELISA according to the manufacturer's instructions (PakAutoAssay, Immucor GTI Diagnostic, Inc, USA). ${ }^{37}$

\section{4 | Statistical analyses}

This study was exploratory and not powered to address any predefined hypothesis. Safety was assessed using the safety analysis set. The TEAEs were described for each treatment arm by preferred term and system organ class according to the Medical Dictionary for Regulatory Authorities version 19.1. Efficacy was assessed using the full analysis set, defined as all randomized patients with at least one post-baseline primary efficacy observation (platelet count result). Efficacy analysis followed the intent-to-treat principle. Patients receiving rescue medication were excluded from the efficacy and PD analyses from the day of rescue. Data until the last visit of the first cycle of the open-label treatment period are reported.

\section{3 | RESULTS}

\section{1 | Patient disposition, demographics, and baseline characteristics}

Sixty-two patients were screened, of whom 38 were randomized 1:1:1 to receive four weekly intravenous infusions of placebo $(N=12)$ or efgartigimod at a dose of $5 \mathrm{mg} / \mathrm{kg}(\mathrm{N}=13)$ or $10 \mathrm{mg} / \mathrm{kg}(\mathrm{N}=13)$ (Figure S2). The most common reasons for screening failure included the prior use of prohibited medication, an active infection or a recent serious infection within eight weeks prior to screening, and clinically significant laboratory abnormalities at screening. Overall, 35 (92.1\%) patients completed the treatment period. Twelve (31.6\%) patients who relapsed during the 21-week follow-up period, entered the openlabel treatment period, and received four weekly intravenous infusions of efgartigimod at $10 \mathrm{mg} / \mathrm{kg}$. Among these 12 patients, two (16.7\%) received efgartigimod at $5 \mathrm{mg} / \mathrm{kg}$ in the double-blind period, six (50.0\%) received efgartigimod at $10 \mathrm{mg} / \mathrm{kg}$, and four (33.3\%) received placebo.

Study demographics and baseline characteristics were generally comparable across the treatment groups (Table 1). Twenty-eight (73.7\%) patients were classified as chronic (more than 12 months from diagnosis), eight (21.1\%) as persistent (between 3-12 months from diagnosis), and two (5.3\%) as newly diagnosed (within 3 months of diagnosis). Median duration of ITP was 4.82 years (range $0.1-47.8$ ). Twenty (52.6\%) patients had baseline platelet count $<15 \times 10^{9} / \mathrm{L}$. The median number of prior ITP treatments was 2.0 (0-10). Nine patients (23.7\%) had previously received rituximab, 14 (36.8\%) a TPO-RA of whom 10 were continuing a TPO-RA at baseline, and six (15.8\%) had prior splenectomy. Twenty-seven (71.1\%) patients were receiving at least one concurrent ITP therapy at baseline, and in spite of this, still had qualifying platelet counts $<30 \times 10^{9} / \mathrm{L}$.

\section{2 | Clinical pharmacology}

Efgartigimod at 5 and $10 \mathrm{mg} / \mathrm{kg}$ induced a rapid reduction of total lgG levels (Figure S3), up to a maximum mean change of $60.4 \%$ on efgartigimod $5 \mathrm{mg} / \mathrm{kg}$ (from $9.9 \mathrm{~g} / \mathrm{L}[\mathrm{SD}=3.2$ ] at baseline to $4.0 \mathrm{~g} / \mathrm{L}$ [SD = 0.8]) and $63.7 \%$ on $10 \mathrm{mg} / \mathrm{kg}$ (from $10.6 \mathrm{~g} / \mathrm{L}[\mathrm{SD}=5.1$ ] at baseline to $4.1 \mathrm{~g} / \mathrm{L}[S D=2.0]$ ) three days after the fourth infusion, while IgG levels in the placebo group remained unchanged. IgG reduction was observed for all four IgG subtypes (Figure S4). Mean percentage changes from baseline of $\lg A$, $\lg D$, IgE, and $\lg M$, as well as of albumin, were similar between placebo and the efgartigimod treated groups, mostly within $\pm 10 \%-15 \%$ of baseline (data not shown) and changes were not considered clinically relevant.

Pre-dose anti-drug antibodies (ADAs) were detectable in one (7.7\%) patient treated with efgartigimod at $5 \mathrm{mg} / \mathrm{kg}$, three $(23.1 \%)$ with efgartigimod at $10 \mathrm{mg} / \mathrm{kg}$, and two (16.7\%) with placebo. Postdose ADA titers were detected in five (38.5\%) patients treated with efgartigimod at $5 \mathrm{mg} / \mathrm{kg}$, four (30.8\%) with efgartigimod at $10 \mathrm{mg} / \mathrm{kg}$, and two (16.7\%) with placebo. Positive ADA titers, measured in all groups, did not have an apparent effect on PK/PD parameters. Analysis of the antiplatelet antibody eluates revealed the presence of platelet-associated autoantibodies (GPIIb/IIla, GPIb/IX, and GPla/Ila) in all randomized patients. In $8 / 12$ (66.7\%) patients treated with efgartigimod at $5 \mathrm{mg} / \mathrm{kg}$, and $7 / 10(70.0 \%)$ treated with efgartigimod at $10 \mathrm{mg} / \mathrm{kg}$, a reduction greater than $40 \%$ in the platelet-associated autoantibody signal for at least one type of autoantibody was observed at days $25 / 29$ and/or 78 . One (7.7\%) patient in the $5 \mathrm{mg} / \mathrm{kg}$ group and three $(23.1 \%)$ in the $10 \mathrm{mg} / \mathrm{kg}$ group were not evaluable either due to a missing baseline sample, or because all post-dose samples were obtained after rescue therapy. The presence of autoantibodies in serum was less prevalent.

\section{3 | Safety}

Nine (69.2\%) patients treated with efgartigimod at $5 \mathrm{mg} / \mathrm{kg}$, 11 (84.6\%) with efgartigimod at $10 \mathrm{mg} / \mathrm{kg}$, and seven (58.3\%) with placebo experienced at least one TEAE, which were mainly mild or moderate in severity (Table 2 ). No deaths were reported. No clinically relevant changes in vital signs, electrocardiogram parameters, physical examination, and clinical laboratory assessments (eg, albumin) were observed. One (7.7\%) patient treated with efgartigimod at $10 \mathrm{mg} / \mathrm{kg}$ experienced a worsening of ITP leading to drug discontinuation. This serious TEAE was the only TEAE with CTCAE severity grade 4 (ie, life threatening) and was considered unlikely related to efgartigimod. One (7.7\%) patient treated with efgartigimod at $10 \mathrm{mg} / \mathrm{kg}$ and two (16.7\%) treated with placebo, experienced at least one TEAE that was related to treatment in the opinion of the investigator. Among the 12 patients who entered the open-label treatment period, seven (58.3\%) patients experienced at least one TEAE, and none were considered treatmentrelated TEAEs. Two serious TEAEs considered unrelated to efgartigimod were reported for two (16.7\%) patients (pneumonia and endometrial polyposis). No bleeding TEAEs were deemed study drugrelated. 
TABLE 1 Summary of demographics and baseline characteristics

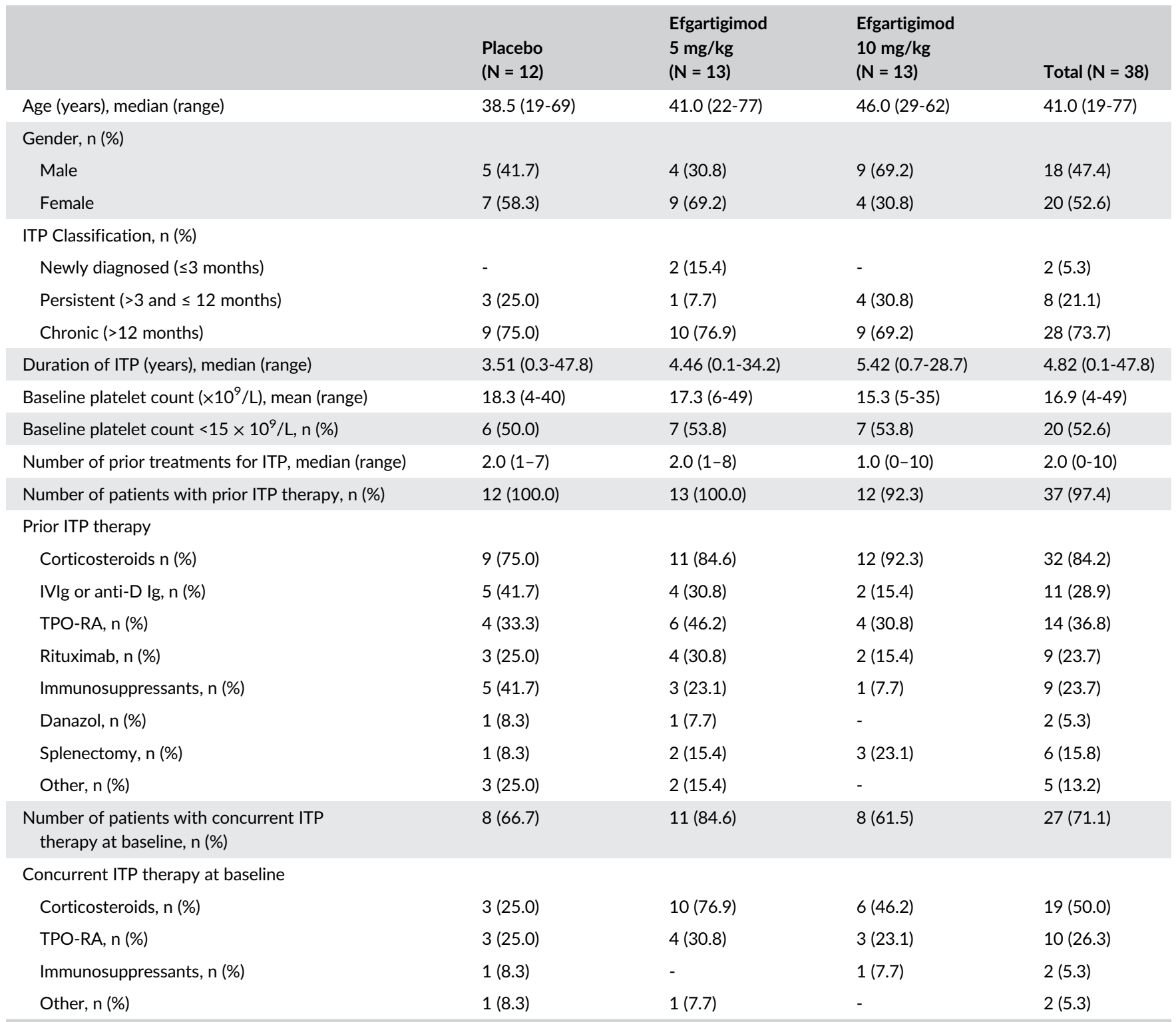

Note: percentages are based on $\mathrm{N}$.

Abbreviations: Ig, immunoglobulin; ITP, immune thrombocytopenia, IVIg, intravenous immunoglobulin; N, number of patients in the analysis set; $\mathrm{n}$, observed number of patients within each treatment group; TPO-RA, thrombopoietin receptor agonist.

\section{4 | Efficacy}

Both efgartigimod-treated groups achieved a higher maximum mean platelet count change from baseline compared to the placebo group (Figure S5). A platelet count of $\geq 50 \times 10^{9} / \mathrm{L}$ at any time was achieved by seven (53.8\%) patients in both efgartigimod-treated groups, and six (50.0\%) in the placebo group (Figure 1). A platelet count $\geq 100 \times 10^{9} / \mathrm{L}$ at any time was achieved by six (46.2\%) patients in the efgartigimod $5 \mathrm{mg} / \mathrm{kg}$ group, five (38.5\%) in the efgartigimod $10 \mathrm{mg} / \mathrm{kg}$ group, and one (8.3\%) in the placebo group. The International Working Group definition of "response" (platelet count $\geq 30 \times 10^{9} / \mathrm{L}$ and $<100 \times 10^{9} / \mathrm{L}$, and at least doubling of baseline platelet count confirmed on at least two separate consecutive occasions $\geq 7$ days apart, and the absence of bleeding) and "complete response" (platelet count $\geq 100 \times 10^{9} / \mathrm{L}$ confirmed on at least two separate consecutive occasions $\geq 7$ days apart, and the absence of bleeding) was achieved by five (38.5\%) patients in the efgartigimod $5 \mathrm{mg} / \mathrm{kg}$ group, five (38.5\%) in the efgartigimod $10 \mathrm{mg} / \mathrm{kg}$ group, and two (16.7\%) in the placebo group. Two newly diagnosed patients with ITP in the efgartigimod $5 \mathrm{mg} / \mathrm{kg}$ group, and one chronic patient in the efgartigimod $10 \mathrm{mg} / \mathrm{kg}$ group had a sustained response throughout the follow-up period (up to day 162).

Post hoc analyses were performed to further characterize the magnitude and duration of effect of efgartigimod (Figure 1). A platelet count $\geq 50 \times 10^{9} / \mathrm{L}$ on at least two occasions was 
TABLE 2 Summary of treatment emergent adverse events

\begin{tabular}{|c|c|c|c|}
\hline Main study & $\begin{array}{l}\text { Placebo } \\
(N=12) n(\%)\end{array}$ & $\begin{array}{l}\text { Efgartigimod } \\
5 \mathrm{mg} / \mathrm{kg} \\
(\mathrm{N}=13) \mathrm{n}(\%)\end{array}$ & $\begin{array}{l}\text { Efgartigimod } \\
10 \mathrm{mg} / \mathrm{kg} \\
(\mathrm{N}=13) \mathrm{n}(\%)\end{array}$ \\
\hline Patients with at least 1 TEAE & $7(58.3)$ & $9(69.2)$ & $11(84.6)$ \\
\hline Patients with at least 1 treatment-related TEAE & $2(16.7)$ & - & $1(7.7)$ \\
\hline Patients with at least 1 serious TEAE & - & - & $1(7.7)$ \\
\hline Purpura & - & $2(15.4)$ & $1(7.7)$ \\
\hline Ecchymosis & - & $1(7.7)$ & $1(7.7)$ \\
\hline Rash & - & $1(7.7)$ & $1(7.7)$ \\
\hline Hematoma & - & $3(23.1)$ & $2(15.4)$ \\
\hline Cystitis & - & $1(7.7)$ & $1(7.7)$ \\
\hline Productive cough & $1(8.3)$ & $1(7.7)$ & - \\
\hline Headache & $2(16.7)$ & $1(7.7)$ & - \\
\hline Open-label treatment period & \multicolumn{3}{|c|}{ Efgartigimod $10 \mathrm{mg} / \mathrm{kg}(\mathrm{N}=12) \mathrm{n}(\%)$} \\
\hline Patients with at least $1 \mathrm{TEAE}$ & \multicolumn{3}{|c|}{$7(58.3 \%)$} \\
\hline Patients with at least 1 treatment-related TEAE & \multicolumn{3}{|c|}{-} \\
\hline Patients with at least 1 serious TEAE & \multicolumn{3}{|c|}{$2(16.7)$} \\
\hline \multicolumn{4}{|l|}{ Most common TEAEs (reported in $\geq 2$ patients overall) } \\
\hline Alanine aminotransferase increased & \multicolumn{3}{|c|}{$2(16.7)$} \\
\hline
\end{tabular}

Abbreviations: $\mathrm{N}$, number of patients in the analysis set; $\mathrm{n}$, number of patients with event within each treatment group under safety analysis set; TEAE, treatment emergent adverse event.

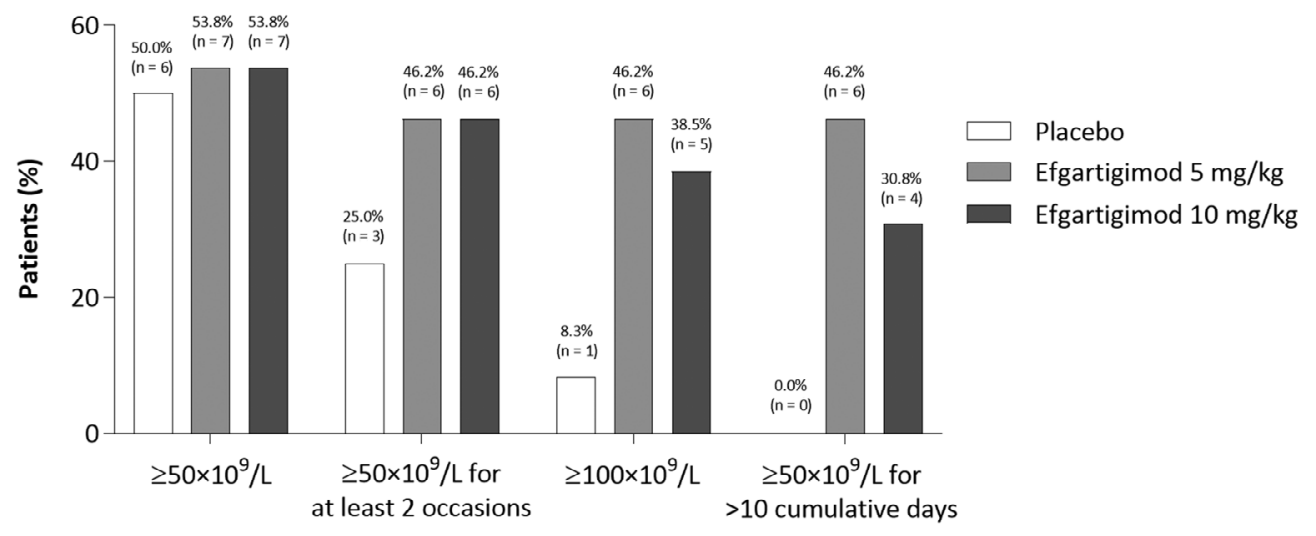

FIGURE 1 Proportion of patients achieving increasing thresholds of platelet count assessed per treatment group during the main study. Patients receiving rescue medication were excluded from the analysis from the day of rescue. $n$, number of patients achieving the threshold

Platelet count threshold

achieved by six (46.2\%) patients in both efgartigimod-treated groups, and three (25.0\%) in the placebo group. For these patients, the mean cumulative duration of platelet count $\geq 50 \times 10^{9} / \mathrm{L}$ was 24.5 days $(S D=20.70$ ), ranging between three and 73 days for efgartigimod-treated patients, and 7.3 days $(S D=2.89)$, ranging between four and nine days for placebotreated patients. Additionally, 10 (38.5\%) efgartigimod-treated patients (six and four in the efgartigimod 5 and $10 \mathrm{mg} / \mathrm{kg}$ groups, respectively) and zero (0.0\%) placebo-treated patients achieved a platelet count $\geq 50 \times 10^{9} / \mathrm{L}$ for a cumulative duration of more than 10 days. The first time of achieving a platelet count $\geq 50 \times 10^{9} / \mathrm{L}$ (for patients achieving a platelet count $\geq 50 \times 10^{9} / \mathrm{L}$ on at least two occasions) ranged from eight to 43 days for the efgartigimodtreated patients. 
FIGURE 2 Mean platelet count \pm SEM $\left(\times 10^{9} /\right.$ L, circles), mean percentage change from baseline of total IgGs \pm SEM (triangles), and percentage of patients with total $\mathrm{WHO}$ score $>0$ (squares) assessed per treatment group during the main study. (A) Placebo,

(B) efgartigimod $5 \mathrm{mg} / \mathrm{kg}$, and (C) efgartigimod $10 \mathrm{mg} / \mathrm{kg}$. Patients receiving rescue medication were excluded from the analysis from the day of rescue (as indicated in the table below the figure). Arrows on the $\mathrm{X}$-axis indicate time points of treatment administration
(A)

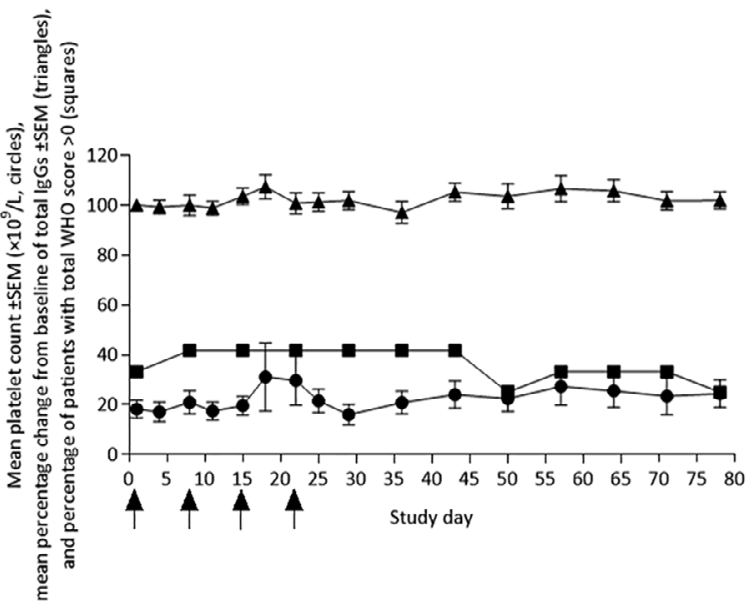

(B)

(C)

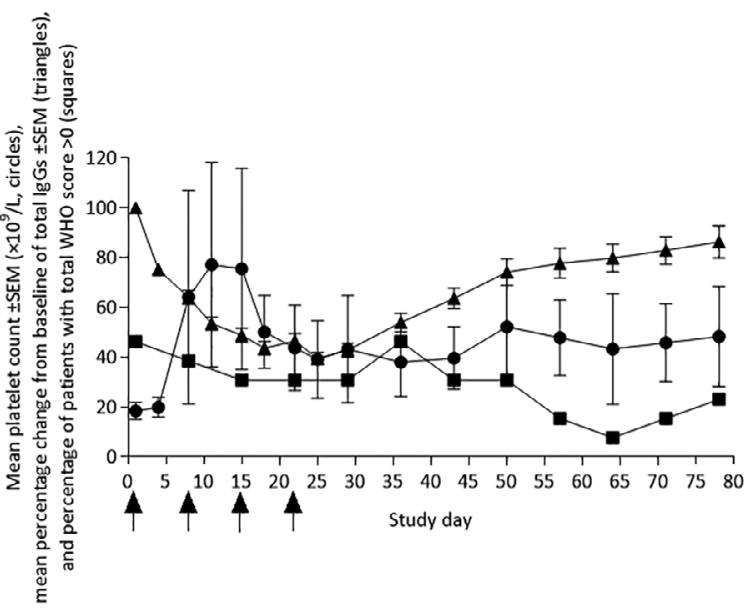

\begin{tabular}{|c|c|c|c|c|c|c|c|c|}
\hline Study day & 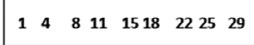 & 36 & 43 & 50 & 57 & 64 & 71 & 78 \\
\hline Placebo, n & $12 \quad 1212 \quad 12 \quad 1212 \quad 1212 \quad 12$ & 12 & 11 & 9 & 8 & 8 & 8 & 7 \\
\hline Efgartigimod 5 mg/kg, n & 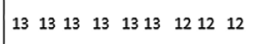 & 12 & 11 & 10 & 9 & 9 & 9 & 9 \\
\hline Efgartigimod $10 \mathrm{mg} / \mathrm{kg}, \mathrm{n}$ & 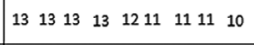 & 10 & 10 & 10 & 10 & 10 & 10 & 10 \\
\hline
\end{tabular}

Four (30.8\%) patients in the efgartigimod $5 \mathrm{mg} / \mathrm{kg}$ group received rescue treatment during the double-blind period, three (23.1\%) had not achieved a platelet count $\geq 50 \times 10^{9} / \mathrm{L}$. Three (23.1\%) patients received rescue therapy in the efgartigimod $10 \mathrm{mg} / \mathrm{kg}$ group during the double-blind period, two of whom had only received three doses.
None of the patients had achieved a platelet count $\geq 50 \times 10^{9} / \mathrm{L}$. One placebo patient received rescue therapy at day 53 .

Of the 12 patients who entered the open-label treatment period and received an additional cycle of four weekly infusions of efgartigimod $10 \mathrm{mg} / \mathrm{kg}$, three (25.0\%) had achieved platelet counts 
$\geq 50 \times 10^{9} / \mathrm{L}$ on at least two occasions during the double-blind period (Table S1). Eight out of 12 (66.7\%) patients achieved platelet counts $\geq 50 \times 10^{9} / \mathrm{L}$ on at least two occasions in the open-label treatment period. Among these eight patients, two from the efgartigimod group at $5 \mathrm{mg} / \mathrm{kg}$, and three from the placebo group had not responded in the double-blind period. Three patients re-treated with efgartigimod at $10 \mathrm{mg} / \mathrm{kg}$ reached this threshold during the double-blind period and the open-label treatment period.

\section{5 | Bleeding-related events}

The incidence, location and severity of any bleeding symptoms were recorded using the World Health Organization (WHO) bleeding scale, and the ITP-specific bleeding assessment tool (ITP-BAT) (Figures S6A and $\mathrm{S} 6 \mathrm{~B}$, respectively). ${ }^{38}$ The proportion of patients with bleeding (total WHO >0) decreased in both efgartigimod 5 and $10 \mathrm{mg} / \mathrm{kg}$ groups, from $46.2 \%$ at baseline to a minimum of $7.7 \%$ at day 64 , and from $38.5 \%$ at baseline to a minimum of $7.7 \%$ at day 29 , respectively (all timepoints shown in Figure 2). In the placebo group, the proportion of patients with bleeding decreased from $33.3 \%$ at baseline to a minimum of $25.0 \%$ at day 50 .

\section{4 | DISCUSSION}

This randomized, double-blind, placebo-controlled Phase 2 study assessed the safety and efficacy of efgartigimod in patients with predominantly longstanding ITP (median disease duration of 4.82 [0.1-47.8] years), who had an insufficient response to prior ITP therapy and/or splenectomy. Twenty (52.6\%) patients had a baseline platelet count $<15 \times 10^{9} / \mathrm{L}$.

Efgartigimod was well tolerated with no dose-related safety observations and the safety profile was consistent with previous observations in healthy volunteers and myasthenia gravis patients. ${ }^{33,36}$ No increased risk of infection was apparent in the efgartigimod-treated groups compared to the placebo group. One patient with a history of splenectomy had pneumonia in the open-label treatment period, occurring eight weeks after the last dose of efgartigimod, when total IgG levels were approaching baseline levels. The investigator considered this TEAE not related to treatment.

Targeting FcRn with efgartigimod resulted in rapid and selective IgG reduction, and a greater numerical reduction was observed in the efgartigimod $10 \mathrm{mg} / \mathrm{kg}$ group, without impacting the levels of other immunoglobulin isotypes. Additionally, the total IgG reduction did not reach the low thresholds previously reported to be associated with increased risk of infection in diseases causing hypogammaglobulinemia. ${ }^{39}$ Notably, efgartigimod administration did not result in a reduction of albumin levels, which has been observed with some anti-FcRn monoclonal antibodies, ${ }^{40,41}$ suggesting that the Fc fragment efgartigimod is not interfering with albumin binding or influencing the fate of $\mathrm{FCRn} .{ }^{33}$ Autoantibodies were identified in all patients in this study and were generally reduced following efgartigimod treatment. However, no apparent correlation with the extent of the clinical effect could be observed, which could possibly be due to the small sample size and the inherent autoantibody assay limitations in ITP. ${ }^{42}$

Efgartigimod-treated groups achieved a higher maximum mean platelet count change from baseline compared to the placebo group. The early and substantial increase in the efgartigimod $5 \mathrm{mg} / \mathrm{kg}$ group could be explained by one patient who was receiving a stable dose of TPO-RA (eltrombopag) as concurrent ITP therapy, and whose platelet count increased to more than $500 \times 10^{9} / \mathrm{L}$ from day 8 to 15 . It will be interesting to further investigate whether there is a synergistic effect of IgG-depletion by efgartigimod and other ITP treatments with different mechanisms of action, such as the TPO-RA. At later time points, the distribution of platelet counts in both efgartigimod-treated groups generally tended to be higher than in the placebo group, especially in the efgartigimod $10 \mathrm{mg} / \mathrm{kg}$ group.

A high number of patients receiving placebo achieved a single platelet count $\geq 50 \times 10^{9} / \mathrm{L}$ during the study (6 [50\%] compared to, for example, $14 \%$ across two, 24-week fostamatinib Phase 3 studies). ${ }^{13,43}$ However, post hoc analyses requiring greater frequency or duration of platelet count $\geq 50 \times 10^{9} / \mathrm{L}$, or increased platelet count to $\geq 100 \times 10^{9} / \mathrm{L}$, demonstrated the efficacy of efgartigimod. Six patients $(46 \%)$ treated in both efgartigimod groups showed an increase in platelet count $>50 \times 10^{9} / \mathrm{L}$ on at least two occasions. Additionally, substantially more active-treated patients achieved a platelet count $\geq 50 \times 10^{9} / \mathrm{L}$ for more than 10 cumulative days compared to the placebo group (10 [38\%] vs 0 [0\%], respectively).

In this study, a high variability in onset and duration of response was observed following a short exposure to efgartigimod. This could be suggestive of differential contributions of the various pathogenic autoantibody activities across different patients. As exemplified in Figure S7A, some efgartigimod-treated patients showed a rapid increase in platelet counts, reminiscent of response times reported for anti-CD16 antibody therapy, ${ }^{44} \mathrm{IVIg}$ therapy or splenectomy. This suggests that in some patients, a limited reduction of autoantibody levels is sufficient to inhibit Fc gamma receptor-mediated phagocytosis of opsonized platelets by macrophages present in the liver and spleen. Other patients showed a delayed time to response as illustrated in Figure S7B. For those patients, a rise in platelet counts was only observed after the fourth infusion (day 22), which could indicate that either a more profound autoantibody reduction is needed, and/or that the main pathogenic action of the autoantibodies consists of impairing platelet production by the megakaryocytes in the bone marrow. In such a scenario, megakaryocyte recovery would need to take place first before platelet counts can increase. Additionally, some patients demonstrated a double platelet peak following efgartigimod treatment, as exemplified in Figure S7C, suggesting two distinct pathogenic autoantibody mechanisms with different kinetics. Interestingly, this phenomenon was also described in patients with acute ITP treated with plasmapheresis. ${ }^{45}$

Most patients who responded to efgartigimod had a transient increase in platelet counts, with counts returning to baseline levels in the treatment-free follow-up period. Two newly diagnosed and one chronic patient with ITP remained in remission throughout the followup period. Similar observations were made following plasmapheresis 
in patients with acute ITP, ${ }^{29,45}$ but responses for patients with chronic ITP were solely reported to be transient.

Twelve patients (including four patients who received placebo in the double-blind period) who suffered a relapse in the 21-week follow-up period, defined as platelet count $<30 \times 10^{9} / \mathrm{L}$, received efgartigimod at $10 \mathrm{mg} / \mathrm{kg}$ in the open-label treatment period. The results demonstrated the repeatability of efgartigimod-induced platelet increases as three patients in the efgartigimod $10 \mathrm{mg} / \mathrm{kg}$ group who had achieved platelet count $\geq 50 \times 10^{9} / \mathrm{L}$ on at least two occasions during the double-blind period, achieved this threshold again with re-treatment. Interestingly, the two patients who, upon initial treatment with efgartigimod $5 \mathrm{mg} / \mathrm{kg}$, did not show an increase in platelet count, did so when treated with efgartigimod $10 \mathrm{mg} / \mathrm{kg}$ in the open-label treatment period, suggesting the need for the higher dose or longer exposure to efgartigimod.

Examples of efgartigimod-treated patients with apparent efficacy based on an increase in platelet count were observed in subgroup analyses based on ITP classification (newly diagnosed, persistent or chronic ITP), concurrent ITP treatment, or use of TPO-RA. Nevertheless, as anticipated in a study with limited number of patients, there were no clear differences in each of these subcategories.

The observation that efgartigimod increases platelet counts in patients with ITP predominantly refractory to previous lines of ITP therapy, regardless of prior use of ITP therapies (eg, steroids, rituximab, TPO-RA, and splenectomy), supports the central role of pathogenic IgGs in ITP and potential utility of IgG depletion. Patients benefited at both doses tested, further supporting the IgG reduction hypothesis. There were some signals that the $10 \mathrm{mg} / \mathrm{kg}$ dose may be superior, including the absence of newly diagnosed patients in this group, who may more readily respond to treatments. Additionally, two patients in the main study did not receive all four $10 \mathrm{mg} / \mathrm{kg}$ doses, potentially lowering the response rate in this cohort. Furthermore, patients whose platelet counts did not increase with efgartigimod $5 \mathrm{mg} / \mathrm{kg}$ in the main study, had an increased platelet count upon treatment with efgartigimod $10 \mathrm{mg} / \mathrm{kg}$ in the open-label treatment period. Finally, a decreased incidence of bleeding, measured using the bleeding scales (total WHO and ITP-BAT scores $>0$ ), was observed in both efgartigimodtreated groups, with numerically greater reduction in the efgartigimod $10 \mathrm{mg} / \mathrm{kg}$ group.

Limitations of this signal-finding study included the small number of patients and heterogeneity of the recruited patient population which limited the assessment of effect in different patient profiles. Additionally, the treatment intervention was short, making efficacy analyses challenging and undermining assessment of the duration of effect and potential utility as chronic treatment.

To conclude, a short treatment cycle of 4 weekly infusions of efgartigimod in patients with ITP predominantly refractory to previous lines of ITP therapy was well tolerated, markedly reduced IgG levels, was associated with clinically relevant increases in platelet counts in a substantial proportion of patients, and reduced the proportion of patients with bleeding (Figure 2). This suggests that targeted IgG reduction with efgartigimod is a potential new treatment modality in primary ITP and warrants further evaluation of longer-term treatment in a larger Phase 3 study.

\section{ACKNOWLEDGMENTS}

The authors thank the study investigators, coordinators, nurses, and patients and their families for their invaluable contributions to this study. This study was sponsored by argenx. We acknowledge Thierry Cousin who was the medical monitor of the study. We also thank Paul A. Imbach for expert input during the preparation of the manuscript.

\section{CONFLICT OF INTEREST}

Adrian C. Newland: consultant for Amgen, Angle, argenx, Dova, Novartis, Ono, Rigel, and Shionogi; received funding from Amgen, Novartis, and Rigel; received honoraria directly from Amgen, Angle, argenx, Dova, Novartis, Ono, Rigel, and Shionogi; and paid expert testimony from argenx and Rigel. Blanca Sánchez-González: received honoraria directly and paid expert testimony from Novartis, Takeda, Amgen, Alexion, Gilead and Shire; and Board of Directors or its advisory committees at Novartis, Takeda, Amgen. László Rejtő, Miklos Egyed, and Nataliya Romanyuk: none. E. Sally Ward: receives funding and royalty payments from argenx, and has equity ownership in argenx. Marc Michel: consultant for Novartis, Amgen, and Rigel. Howard A. Liebman: consultant for argenx, Novartis, Rigel, Pfizer, Dova, and received funding from Janssen and Bristol-Myers. David J. Kuter: consultant for Actelion (Syntimmune), Agios, Alnylam, Amgen, argenx, Bristol Myers Squibb (BMS), Caremark, Daiichi Sankyo, Dova, Kyowa-Kirin, Merck Sharp Dohme, Momenta, Novartis, Pfizer, Platelet Disorder Support Association, Principia, Protalex, Protalix, Rigel, Sanofi, Genzyme, Shionogi, Shire, Takeda (Bioverativ), UCB, Up-To-Date, Zafgen; and received funding from Actelion (Syntimmune), Agios, Alnylam, Amgen, argenx, Bristol Myers Squibb (BMS), Kezar, Principia, Protalex, Rigel, and Takeda (Bioverativ). Katrien Verschueren: consultant for argenx. Marie Godar, Domenica Gandini, Peter Ulrichts, Jon Beauchamp, Torsten Dreier, Hans de Haard, and Nicolas Leupin: employees and equity ownership in argenx.

\section{AUTHOR CONTRIBUTIONS}

A.C.N., P.U., T.D., M.M., H.A.L., H.H., N.L., and D.J.K. designed the study; A.C.N., B.S.-G., R.L., M.E., and N.R. collected data; A.C.N., M.G., K.V., D.G., P.U., J.B., T.D., M.M., H.A.L., H.H., N.L., and D.J.K. interpreted the data and compiled them for analysis; K.V.: performed statistical analysis; and all authors participated in decisions related to the manuscript content and publication, had access to the primary data, and vouch for the completeness and accuracy of the data and analyses. 


\section{ORCID}

Marie Godar (D) https://orcid.org/0000-0002-5477-7726

E. Sally Ward (iD https://orcid.org/0000-0003-3232-7238

Hans de Haard (D) https://orcid.org/0000-0001-8707-0084

David J. Kuter (D) https://orcid.org/0000-0002-7855-2409

\section{REFERENCES}

1. Rodeghiero F, Stasi R, Gernsheimer T, et al. Standardization of terminology, definitions and outcome criteria in immune thrombocytopenic purpura of adults and children: report from an international working group. Blood. 2009;113(11):2386-2393.

2. Kistangari G, McCrae KR. Immune thrombocytopenia. Hematol Oncol Clin North Am. 2013;27(3):495-520.

3. Neunert C, Lim W, Crowther M, Cohen A, Solberg L, Crowther MA. The American Society of Hematology 2011 evidence-based practice guideline for immune thrombocytopenia. Blood. 2011;117(16):4190-4207.

4. Zufferey A, Kapur R, Semple J. Pathogenesis and therapeutic mechanisms in immune thrombocytopenia (ITP). J Clin Med. 2017;6(2):E16.

5. Cohen YC, Djulbegovic B, Shamai-Lubovitz O, Mozes B. The bleeding risk and natural history of idiopathic thrombocytopenic purpura in patients with persistent low platelet counts. Arch Intern Med. 2000; 160(11):1630-1638.

6. Hill QA, Newland AC. Fatigue in immune thrombocytopenia. $\mathrm{Br} J$ Haematol. 2015;170(2):141-149.

7. McMillan R, Bussel JB, George JN, Lalla D, Nichol JL. Self-reported health-related quality of life in adults with chronic immune thrombocytopenic purpura. Am J Hematol. 2008;83(2):150-154.

8. Snyder CF, Mathias SD, Cella D, Isitt JJ, Wu AW, Young J. Health-related quality of life of immune thrombocytopenic purpura patients: results from a web-based survey. Curr Med Res Opin. 2008;24(10):2767-2776.

9. Doobaree IU, Nandigam R, Bennett D, Newland A, Provan D. Thromboembolism in adults with primary immune thrombocytopenia: a systematic literature review and meta-analysis. Eur J Haematol. 2016;97(4):321-330.

10. Sarpatwari A, Bennett D, Logie JW, et al. Thromboembolic events among adult patients with primary immune thrombocytopenia in the United Kingdom General Practice Research Database. Haematologica. 2010;95(7):1167-1175.

11. Sarpatwari A, Watson S, Erqou $S$, et al. Health-related lifestyle in adults and children with primary immune thrombocytopenia (ITP). $\mathrm{Br}$ J Haematol. 2010;151(2):189-191.

12. Mathias SD, Gao SK, Miller KL, et al. Impact of chronic Immune Thrombocytopenic Purpura (ITP) on health-related quality of life: a conceptual model starting with the patient perspective. Health Qual Life Outcomes. 2008;6(1):13.

13. Bussel J, Arnold DM, Grossbard E, et al. Fostamatinib for the treatment of adult persistent and chronic immune thrombocytopenia: results of two phase 3 , randomized, placebo-controlled trials. Am J Hematol. 2018;93(7):921-930.

14. Cines DB, Blanchette VS. Immune thrombocytopenic purpura. N Engl $J$ Med. 2002;346(13):995-1008.

15. Nugent D, McMillan R, Nichol JL, Slichter SJ. Pathogenesis of chronic immune thrombocytopenia: increased platelet destruction and/or decreased platelet production. Br J Haematol. 2009;146(6):585-596.

16. Stasi R. Immune thrombocytopenia: pathophysiologic and clinical update. Semin Thromb Hemost. 2012;38(5):454-462.

17. Goette NP, Glembotsky AC, Lev PR, et al. Platelet apoptosis in adult immune thrombocytopenia: insights into the mechanism of damage triggered by auto-antibodies. PLoS One. 2016;11(8):e0160563.

18. Najaoui A, Bakchoul T, Stoy J, et al. Autoantibody-mediated complement activation on platelets is a common finding in patients with immune thrombocytopenic purpura (ITP). Eur J Haematol. 2012;88(2):167-174.
19. Li J, van der Wal DE, Zhu G, et al. Desialylation is a mechanism of Fcindependent platelet clearance and a therapeutic target in immune thrombocytopenia. Nat Commun. 2015;6:7737.

20. Houwerzijl EJ, Blom NR, van der Want JJ, et al. Ultrastructural study shows morphologic features of apoptosis and para-apoptosis in megakaryocytes from patients with idiopathic thrombocytopenic purpura. Blood. 2004;103(2):500-506.

21. Chang M, Nakagawa PA, Williams SA, et al. Immune thrombocytopenic purpura (ITP) plasma and purified ITP monoclonal autoantibodies inhibit megakaryocytopoiesis in vitro. Blood. 2003;102(3): 887-895.

22. McMillan R, Wang L, Tomer A, Nichol J, Pistillo J. Suppression of in vitro megakaryocyte production by antiplatelet autoantibodies from adult patients with chronic ITP. Blood. 2004;103(4):13641369.

23. Al-Samkari H, Van Cott EM, Kuter DJ. Platelet aggregation response in immune thrombocytopenia patients treated with romiplostim. Ann Hematol. 2019;98(3):581-588.

24. Middelburg RA, Carbaat-Ham JC, Hesam H, Ragusi MA, Zwaginga JJ. Platelet function in adult ITP patients can be either increased or decreased, compared to healthy controls, and is associated with bleeding risk. Hematology. 2016;21(9):549-551.

25. McMillan R. Antiplatelet antibodies in chronic adult immune thrombocytopenic purpura: assays and epitopes. J Pediatr Hematol Oncol. 2003;25(Suppl 1):S57-S61.

26. McMillan R. Antiplatelet antibodies in chronic immune thrombocytopenia and their role in platelet destruction and defective platelet production. Hematol Oncol Clin North Am. 2009;23(6):1163-1175.

27. Webert KE, Mittal R, Sigouin C, Heddle NM, Kelton JG. A retrospective 11-year analysis of obstetric patients with idiopathic thrombocytopenic purpura. Blood. 2003;102(13):4306-4311.

28. Snyder H, Cochran SK, Balint J, et al. Experience with protein Aimmunoadsorption in treatment-resistant adult immune thrombocytopenic purpura. Blood. 1992;79(9):2237-2245.

29. Blanchette VS, Hogan VA, McCombie NE, et al. Intensive plasma exchange therapy in ten patients with idiopathic thrombocytopenic purpura. Transfusion. 1984;24(5):388-394.

30. Roopenian DC, Akilesh S. FcRn: the neonatal Fc receptor comes of age. Nat Rev Immunol. 2007;7(9):715-725.

31. Challa DK, Velmurugan R, Ober RJ, Sally Ward E. FcRn: from molecular interactions to regulation of IgG pharmacokinetics and functions. Curr Top Microbiol Immunol. 2014;382:249-272.

32. Sand KMK, Bern M, Nilsen J, Noordzij HT, Sandlie I, Andersen JT. Unraveling the interaction between FcRn and albumin: opportunities for design of albumin-based therapeutics. Front Immunol. 2015; 5:682.

33. Ulrichts P, Guglietta A, Dreier T, et al. Neonatal Fc receptor antagonist efgartigimod safely and sustainably reduces IgGs in humans. J Clin Invest. 2018;128(10):4372-4386.

34. Vaccaro C, Zhou J, Ober RJ, Ward ES. Engineering the Fc region of immunoglobulin $\mathrm{G}$ to modulate in vivo antibody levels. Nat Biotechnol. 2005;23(10):1283-1288.

35. Challa DK, Bussmeyer U, Khan T, et al. Autoantibody depletion ameliorates disease in murine experimental autoimmune encephalomyelitis. MAbs. 2013;5(5):655-659.

36. Howard JF, Bril V, Burns TM, et al. Randomized phase 2 study of FcRn antagonist efgartigimod in generalized myasthenia gravis. Neurology. 2019;92(23):e2661-e2673.

37. Cantoni S, Carpenedo M, Nichelatti M, et al. Clinical relevance of antiplatelet antibodies and the hepatic clearance of platelets in patients with immune thrombocytopenia. Blood. 2016;128(17): 2183-2185.

38. Rodeghiero F, Michel M, Gernsheimer T, et al. Standardization of bleeding assessment in immune thrombocytopenia: report from the International Working Group. Blood. 2013;121(14):2596-2606. 
39. Furst DE. Serum immunoglobulins and risk of infection: how low can you go? Semin Arthritis Rheum. 2009;39(1):18-29.

40. Ling LE, Hillson JL, Tiessen RG, et al. M281, an Anti-FcRn antibody: pharmacodynamics, pharmacokinetics, and safety across the full range of IgG reduction in a first-in-human study. Clin Pharmacol Ther. 2019;105(4):1031-1039.

41. Kiessling P, Lledo-Garcia R, Watanabe $S$, et al. The FcRn inhibitor rozanolixizumab reduces human serum IgG concentration: a randomizedphase 1 study. Sci Transl Med. 2017;9(414):eaan1208.

42. Heikal NM, Smock KJ. Laboratory testing for platelet antibodies. Am J Hematol. 2013;88(9):818-821.

43. Cheng G, Saleh MN, Marcher C, et al. Eltrombopag for management of chronic immune thrombocytopenia (RAISE): a 6-month, randomised, phase 3 study. Lancet. 2011;377(9763):393-402.

44. Clarkson SB, Bussel JB, Kimberly RP, Valinsky JE, Nachman RL, Unkeless JC. Treatment of refractory immune thrombocytopenic purpura with an anti-Fcy-receptor antibody. N Engl J Med. 1986;314(19): 1236-1239.
45. Marder V, Nusbacher J, Anderson F. One-year follow-up of plasma exchange therapy in 14 patients with idiopathic thrombocytopenic purpura. Transfusion. 1981;21(3):291-298.

\section{SUPPORTING INFORMATION}

Additional supporting information may be found online in the Supporting Information section at the end of this article.

How to cite this article: Newland AC, Sánchez-González B, Rejtő L, et al. Phase 2 study of efgartigimod, a novel FcRn antagonist, in adult patients with primary immune thrombocytopenia. Am J Hematol. 2020;95:178-187. https:// doi.org/10.1002/ajh.25680 